Supplementary Materials to

\title{
The Jensen Effect and Functional Single Index Models: Estimating the Ecological Implications of Nonlinear Reaction Norms
}

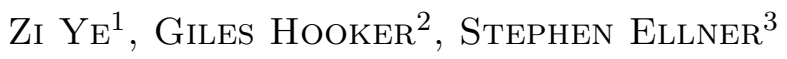

\section{A A Simulated Demonstration}

We present here a brief simulation study to observe the accuracy of curvature estimates. The covariate function $X(t)$ was generated based on a Fourier basis

$$
X_{i}(t)=\mu(t)+\sum_{k=1}^{4} \xi_{i k} \eta_{k}(t), \quad i=1, \cdots, n,
$$

where $\mu(t)=t, \eta_{1}(t)=\frac{1}{\sqrt{2}} \sin (2 \pi t), \eta_{2}(t)=\frac{1}{\sqrt{2}} \cos (2 \pi t), \eta_{3}(t)=\frac{1}{\sqrt{2}} \sin (4 \pi t), \eta_{4}(t)=\frac{1}{\sqrt{2}} \cos (4 \pi t)$, and $\xi_{i k}$ are independent $\mathrm{N}\left(0, \gamma_{k}\right)$ with $\gamma_{1}=1, \gamma_{2}=\frac{1}{2}, \gamma_{3}=\frac{1}{4}, \gamma_{4}=\frac{1}{8}$. The coefficient function is

$$
\beta(t)=\sqrt{2}\left[\frac{1}{\sqrt{12}} \eta_{1}(t)+\frac{1}{\sqrt{12}} \eta_{2}(t)+\frac{1}{\sqrt{6}} \eta_{3}(t)+\frac{1}{\sqrt{6}} \eta_{4}(t)\right] .
$$

We observe that the coefficients for $\beta$ satisfy $\|\boldsymbol{c}\|=1$, under an orthonormal basis. The random errors $\epsilon_{i}$ are simulated as i.i.d. Gaussian noise with mean 0 and $\operatorname{var}(\epsilon)=0.1 \operatorname{var}\left[g\left(\int X \beta\right)\right]$. We selected the sample size as $n=100$ and examined three link functions:

1. $g(s)=e^{-s}$.

2. $g(s)=-s^{2}$.

3. $g(s)=s$.

\footnotetext{
${ }^{1} \mathrm{PhD}$ Candidate, Department of Statistical Science, Cornell University, zy234@cornell.edu

${ }^{2}$ Associate Professor, Department of Statistical Science, Cornell University, and Research School of Finance, Actuarial Studies and Statistics, Australian National University, gjh27@cornell.edu

${ }^{3}$ Professor, Department of Ecology and Evolutionary Biology, Cornell University, spe2@cornell.edu
} 
To measure the performance of our estimators we define the MSE of the estimated $\beta$ and $g^{(k)}$ to be

$$
\mathrm{RSE}=\left[\int(\hat{\beta}(t)-\beta(t))^{2} \mathrm{~d} t\right]^{\frac{1}{2}}
$$

and

$$
\operatorname{RASE}(\mathrm{k})=\left\{\frac{1}{n} \sum_{i=1}^{n}\left[\hat{Y}_{i}^{(k)}-g^{(k)}\left(\int X_{i}(t) \beta(t) \mathrm{d} t\right)\right]^{2}\right\}^{\frac{1}{2}},
$$

where $\hat{Y}_{i}^{(k)}=\hat{g}^{(k)}\left(\int X_{i}(t) \hat{\beta}(t) \mathrm{d} t\right)$ for $k=0,1, \cdots$.

Of particular concern in the results (Table 1) is the substantial discrepancy between estimates from different initial conditions. Ye and Hooker (2018) similarly observed that second derivative estimates were highly sensitive to the effort placed into optimization.

\begin{tabular}{ccc|cc|cc}
\hline & \multicolumn{2}{c}{ g1 } & \multicolumn{2}{c}{ g2 } & \multicolumn{2}{c}{ g3 } \\
\cline { 2 - 7 } Initial & True & equal & True & equal & True & equal \\
\hline RSE & 1.1213 & 0.6417 & 0.5385 & 0.6980 & 0.7608 & 0.7024 \\
RASE(1) & 0.0921 & 0.0800 & 0.0490 & 0.0730 & 0.0706 & 0.0764 \\
RASE(2) & 5.2517 & 3.0516 & 2.9079 & 4.1328 & 5.4393 & 1.2170 \\
\hline
\end{tabular}

Table 1: Simulation results with $\left(\lambda_{g}, \lambda_{\beta}\right)$ selected by GCV. Values in the Table are averages over 100 simulations.

The plots in Figure 1 provide an example of our results. The estimate of the link function nearly overlaps the true curve, indicating that our estimate of the link function is quite accurate. However, for the second derivative, the estimate deviates from the true curve, becoming negative towards the right-hand limit. This reduced accuracy is also evident in the results in Table 1. These plots indicate that our estimate of the curvature is not good enough to use as a basic for decisions on the convexity of $g$. In addition, the performance of the estimators varies a lot from different initial values. In Figure 2 we see that different initial conditions can lead to either over- or under-fitting $g$ ". Further examples are provided in Figures 3 and 4 for quadratic and linear generating $g$ respectively. 

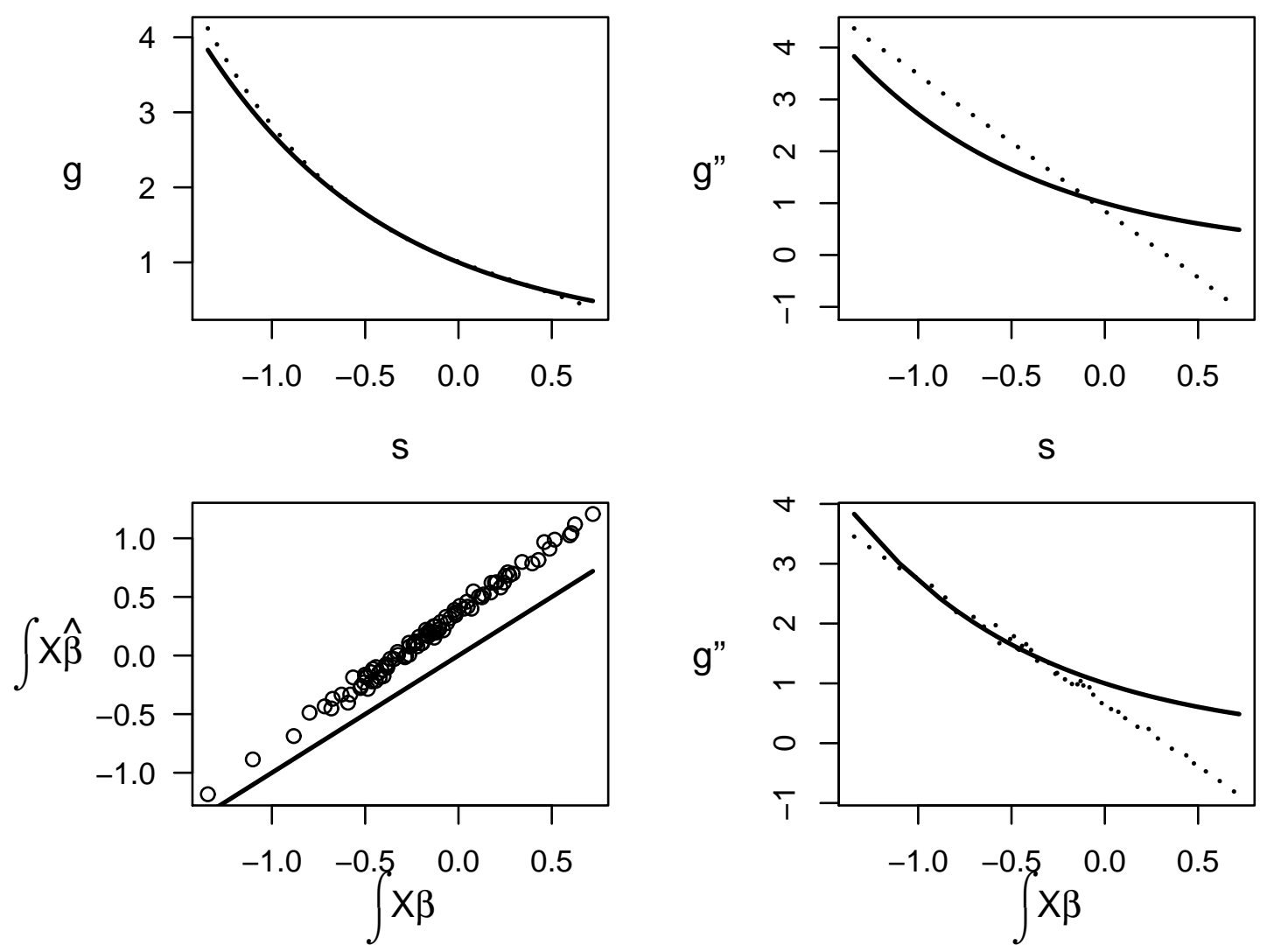

Figure 1: Example estimate for the link function $g(s)=e^{-s}$. Top-left and right panels plot $g$ and $g^{\prime \prime}$ over 1000 equally-spaced grid points between the minimum and maximum of $\int X(t) \hat{\beta}(t) \mathrm{d} t$. Dots are estimated values, and the solid curves are the truth. The bottom-left panel plots $\int X(t) \hat{\beta}(t) \mathrm{d} t$ versus $\int X(t) \beta(t) \mathrm{d} t$ (circles); the solid line is the 1:1 line. The bottom-right panel presents $g^{\prime \prime}$ (black) and $\hat{g}^{\prime \prime}$ (red) evaluated at $\int X(t) \beta(t) \mathrm{d} t$ and $\int X(t) \hat{\beta}(t) \mathrm{d} t$ respectively but plotted against the true argument. 

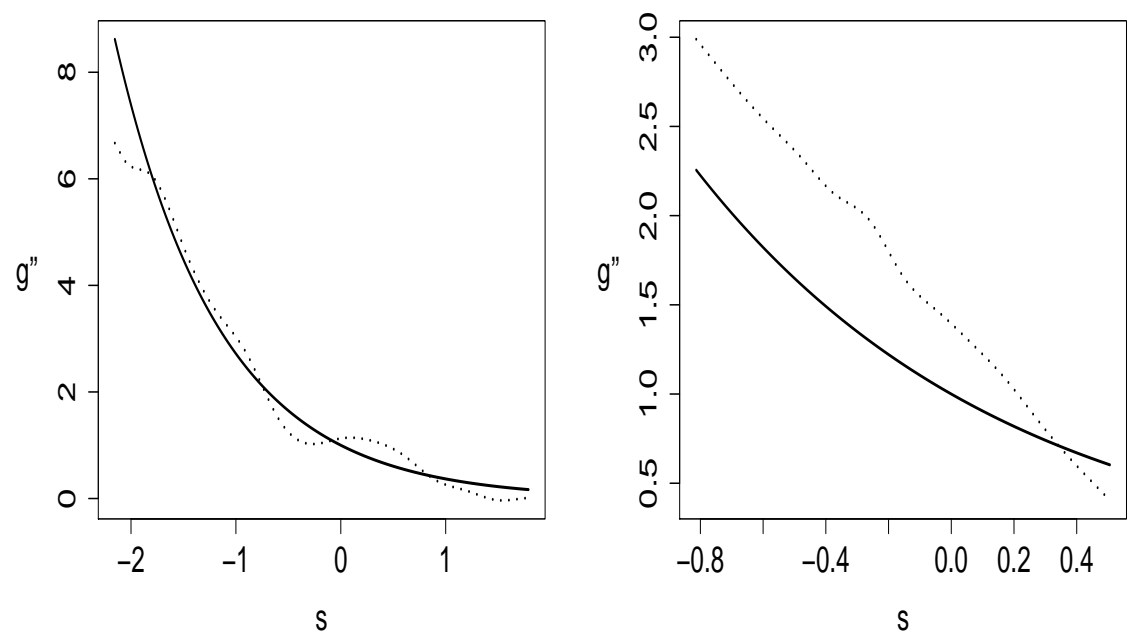

Figure 2: Estimates for $g^{\prime \prime}$ from different initial conditions. Left, using the known true $g$ as the initial condition. Right, starting from equal values of the coefficients. Note that this example was chosen for illustrative purposes and does not use the same data as Figure 1 , the domains of the function are different due to differences in the estimate $\int X_{i}(t) \beta(t) d t$ 

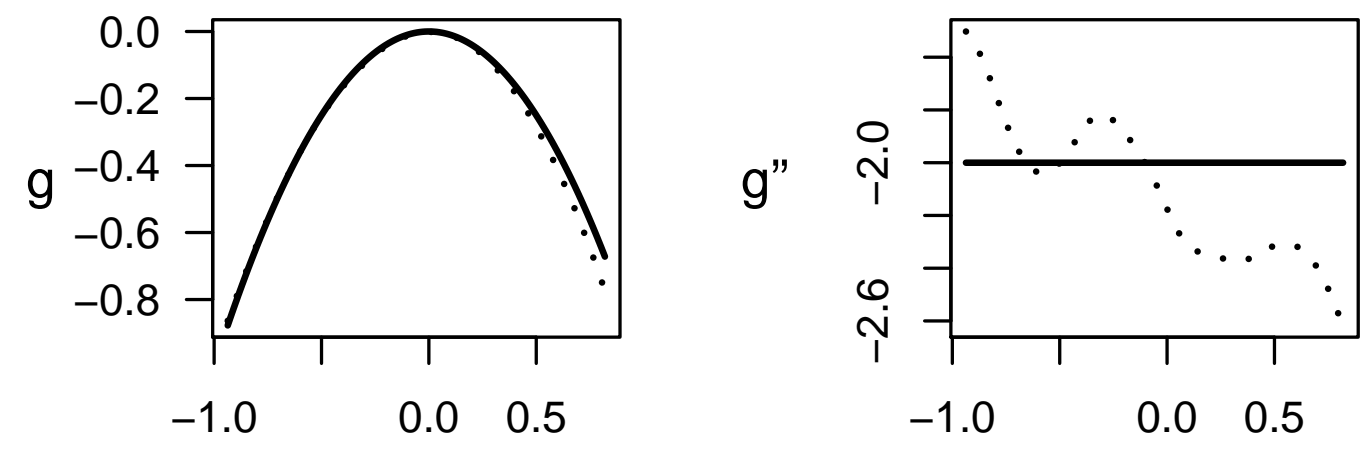

S
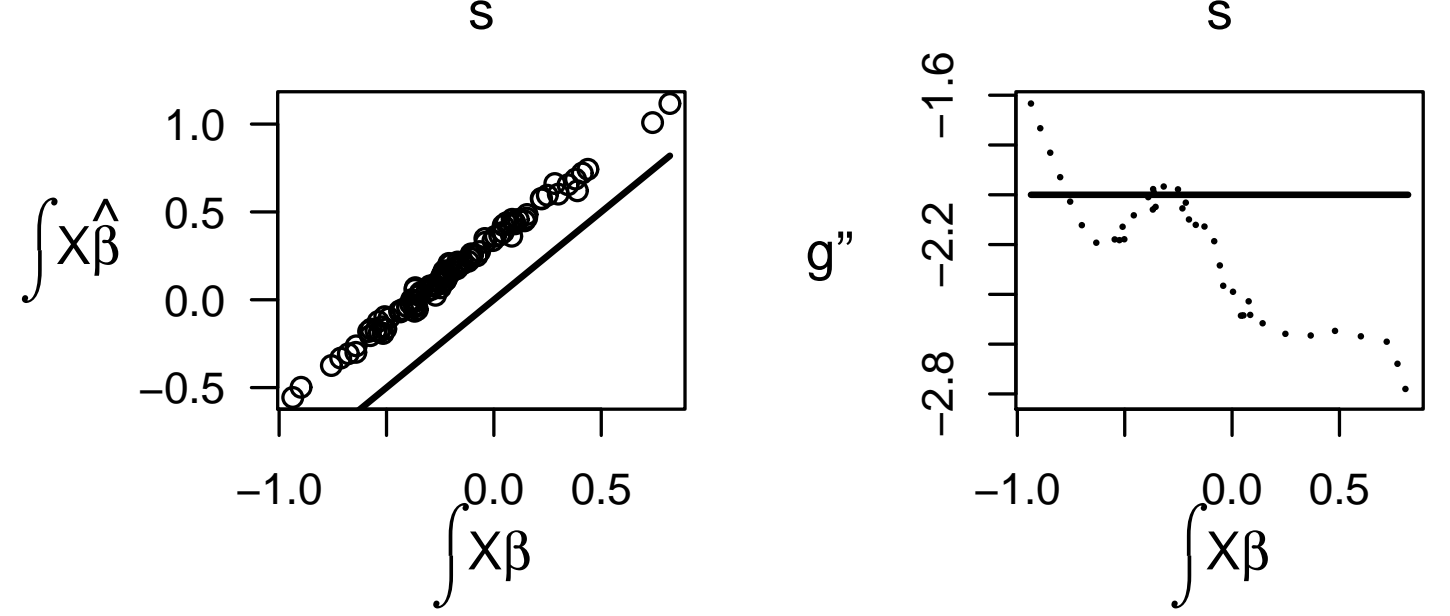

Figure 3: The link function is $g(s)=-s^{2}$. The top-left and right panel are the plots of $g$ and $g$ " over 1000 equally-spaced grid points, while the lower and upper bound are the minimum and maximum of $\int X(t) \hat{\beta}(t) \mathrm{d} t$. The bottom-right panel is the plot of $g$ " over the true $\int X(t) \beta(t) \mathrm{d} t$. The generating model is indicated by solid lines, while dashed lines give the estimated curve. The bottom-left panel is the plot of $\int X(t) \hat{\beta}(t) \mathrm{d} t$ versus $\int X(t) \beta(t) \mathrm{d} t$, with the $y=x$ fit indicated by the solid line. 

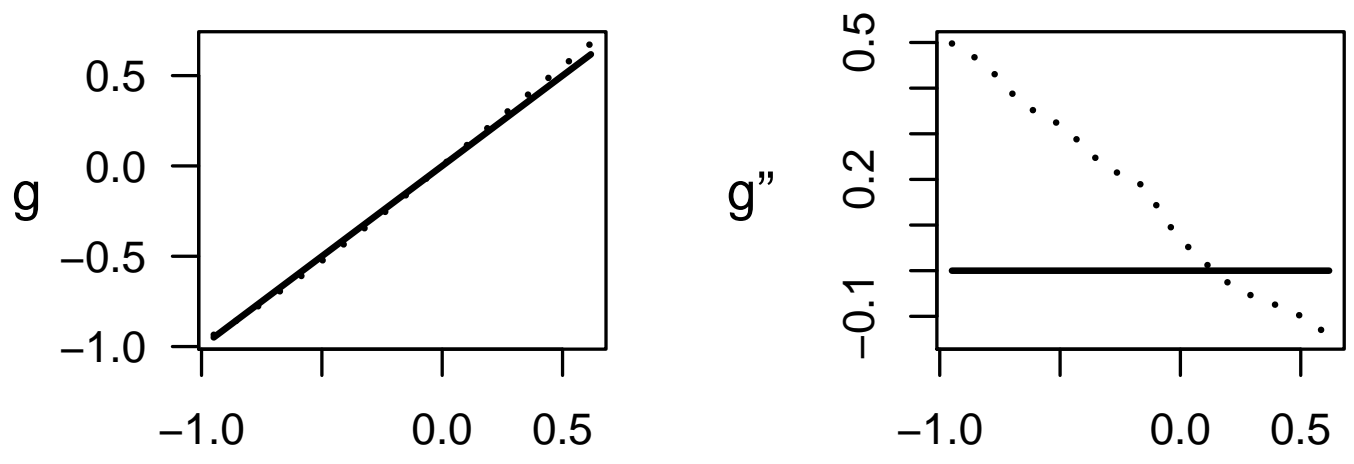

S
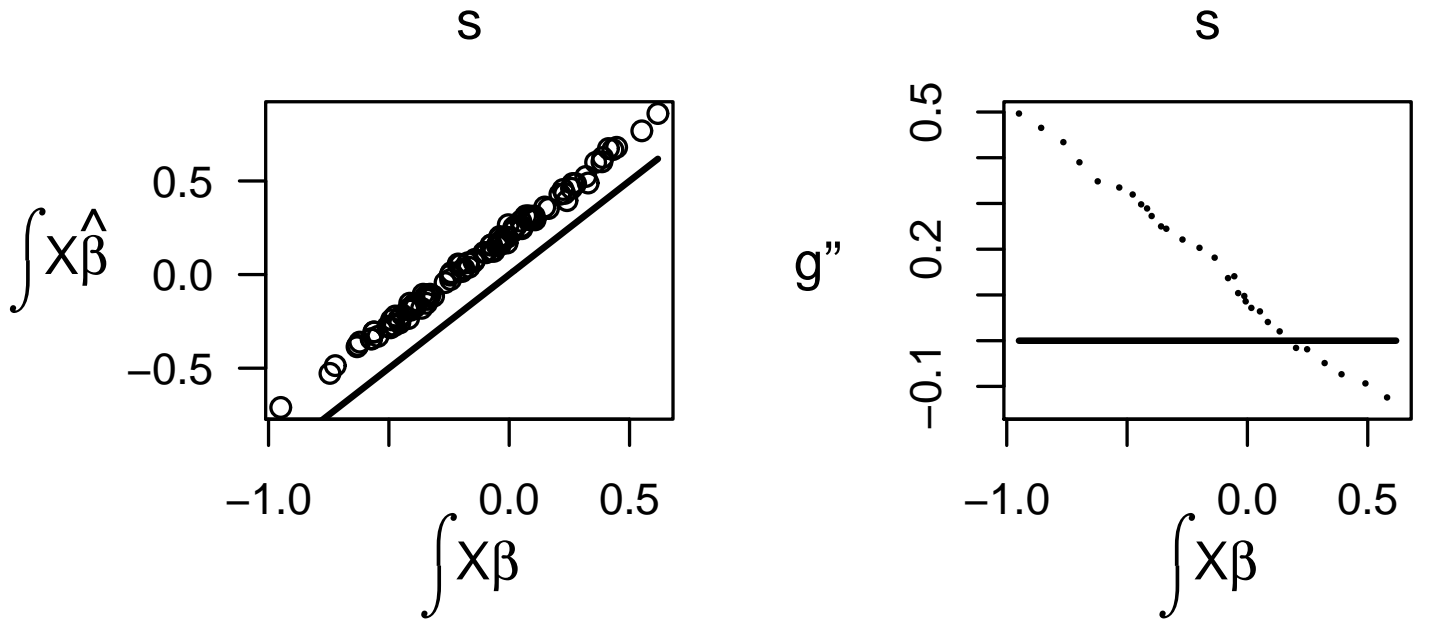

Figure 4: The link function is $g(s)=s$. The top-left and right panel are the plots of $g$ and $g$ " over 1000 equally-spaced grid points, while the lower and upper bound are the minimum and maximum of $\int X(t) \hat{\beta}(t) \mathrm{d} t$. The bottom-right panel is the plot of $g$ " over the true $\int X(t) \beta(t) \mathrm{d} t$. The generating model is indicated by solid lines, while dashed lines give the estimated curve. The bottom-left panel is the plot of $\int X(t) \hat{\beta}(t) \mathrm{d} t$ versus $\int X(t) \beta(t) \mathrm{d} t$, with the $y=x$ fit indicated by the solid line. 

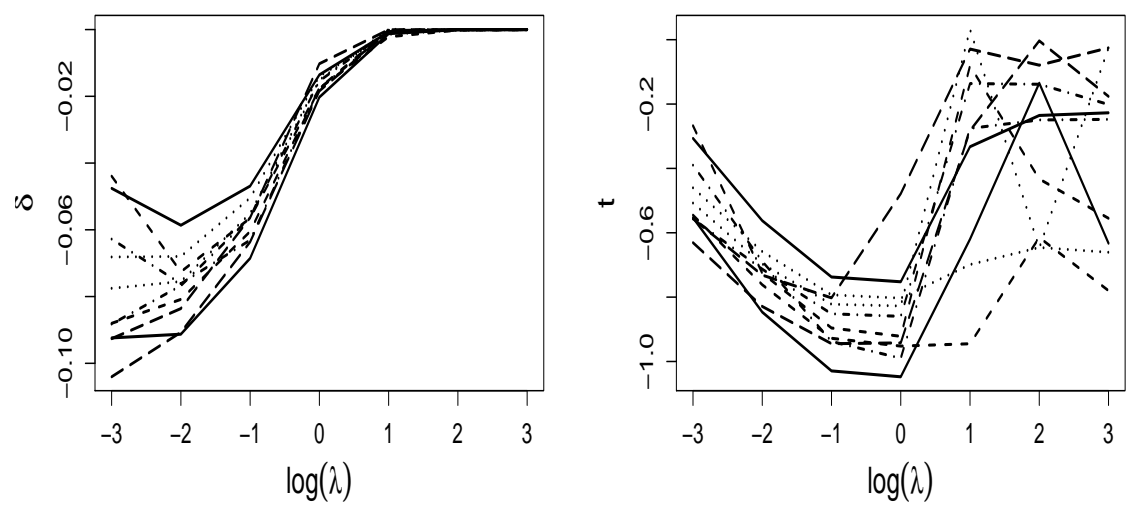

Figure 5: Left: a sample of $\delta_{\lambda}$ as a function of $\lambda$ in a single index model with link function $g(s)=-s^{2}$. Right: the corresponding $t_{\lambda}$ functions.

\section{B Diagnostic Plots for the Jensen Effect: Single Index Model}

Figures 5 and 6 give example $\delta$ functions using a single index model and the corresponding $t$ functions for links $g(s)=-s^{2}$ and $g(s)=s$ respectively.
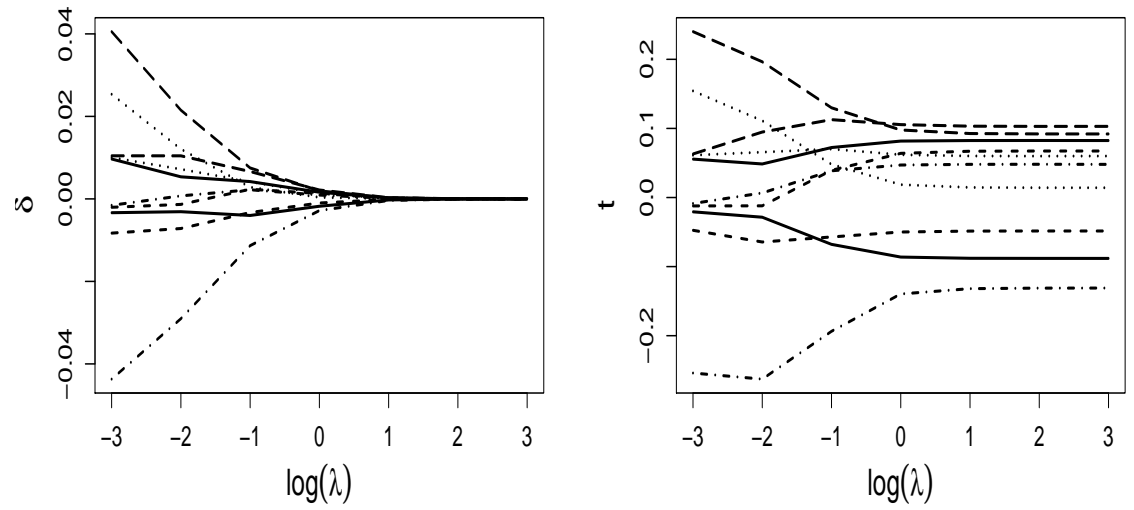

Figure 6: Left: a sample of $\delta_{\lambda}$ as a function of $\lambda$ in a single index model with link function $g(s)=s$. Right: the corresponding $t_{\lambda}$ functions. 


\section{Diagnostic Plots for the Functional Single Index Model}

Figures 7 and 8 give example $\delta$ functions using a single index model and the corresponding $t$ functions for links $g(s)=-s^{2}$ and $g(s)=s$ respectively.
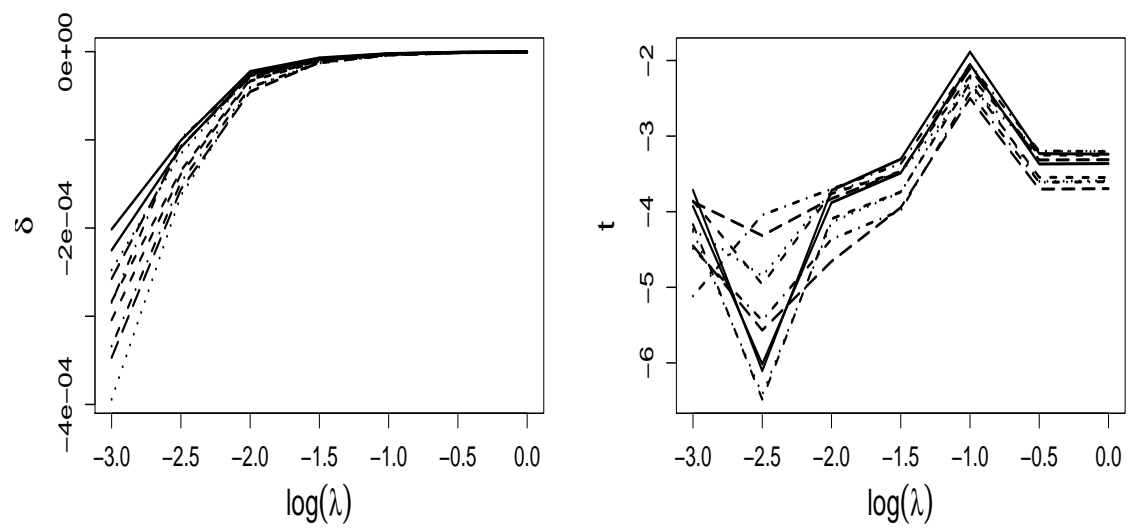

Figure 7: Left: a sample of $\delta_{\lambda}$ as a function of $\lambda$ in a functional single index model with link function $g(s)=-s^{2}$. Right: the corresponding $t_{\lambda}$ functions.
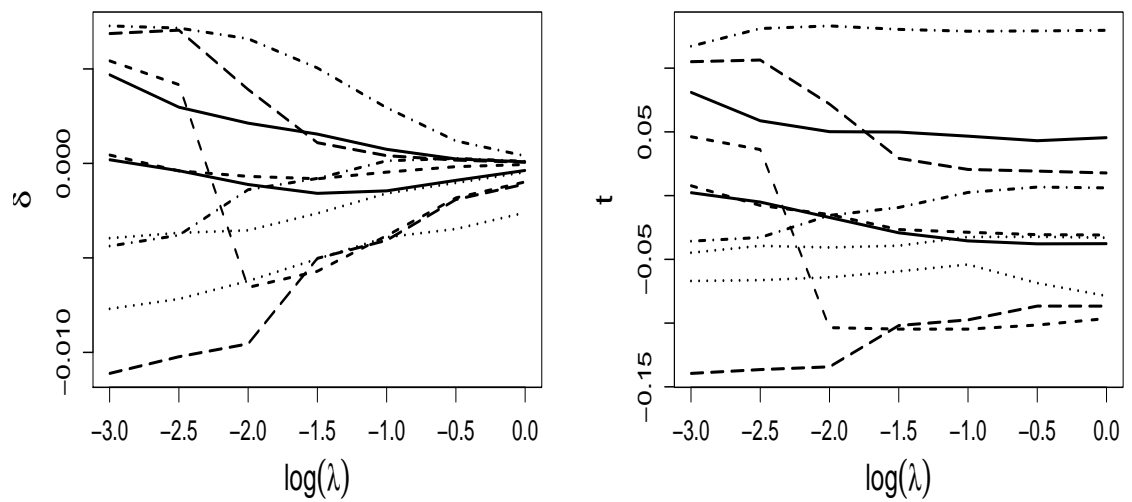

Figure 8: Left: a sample of $\delta_{\lambda}$ as a function of $\lambda$ in a functional single index model with link function $g(s)=s$. Right: the corresponding $t_{\lambda}$ functions. 


\section{On Estimates of Residual Variance}

We use the power simulations in Section 4.3 to confirm our expectation that we can select sigma based on residual squared error at smoothing values selected by GCV. In Figure 9 we examine both single index models and functional single index models. For single index models we plot $\hat{\sigma}_{\lambda}$ as a function of $\lambda$ the first 10 simulations and indicate the value of GCV with an asterisk for each. When the data is generated from a linear relationship, these curves are nearly flat, when we use the maximum value of $\eta$ there are noticeable changes in $\hat{\sigma}_{\lambda}$ but each curve has an extended flat area around the correct value which is reliably estimated by GCV. We note that GCV appropriately chooses large smoothing parameters when the relationship is linear and there is little bias, but selects lower values for simulations with higher curvature. It is less easy to make these plots for functional single index models, and we instead provide a histogram of estimates at the GCV value for both linear and maximally curved relationships where we see that in both cases our estimates focus on approximately the correct values.
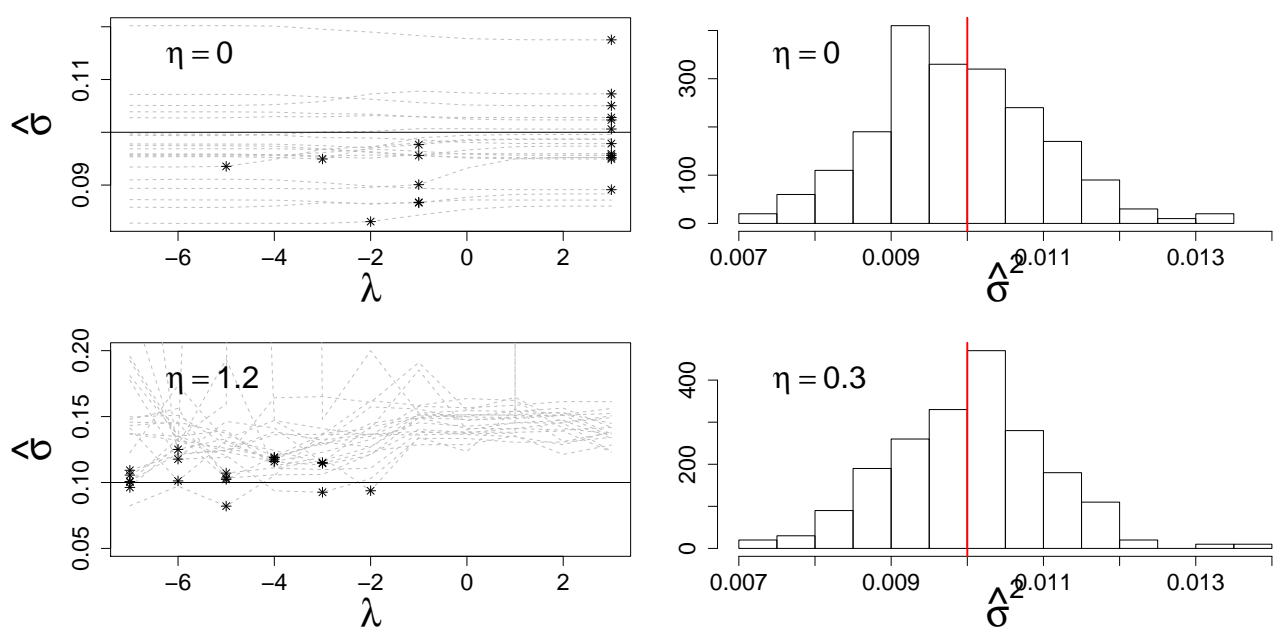

Figure 9: Estimates of residual standard error. Left: for single index models, $\hat{\sigma}_{\lambda}$ as a function of $\lambda$ for the first 10 simulations from a linear relationship (top) and maximally curved (bottom). Right: histograms estimates of $\sigma$ from linear relationships (top) and maximally curved relationships for functional single index models. 


\section{E Plots for the copepod data}

In order to obtain a functional covariate, we smoothed water temperature readings in each lake employing B-splines with 21 knots per year and a second derivative penalty with penalty parameter selected by GCV. These were then evaluated on each of the 60 days prior to a population observation and re-projected onto 12 order 6 Bsplines. Yearly temperature curves for each lake are given in Figure 10

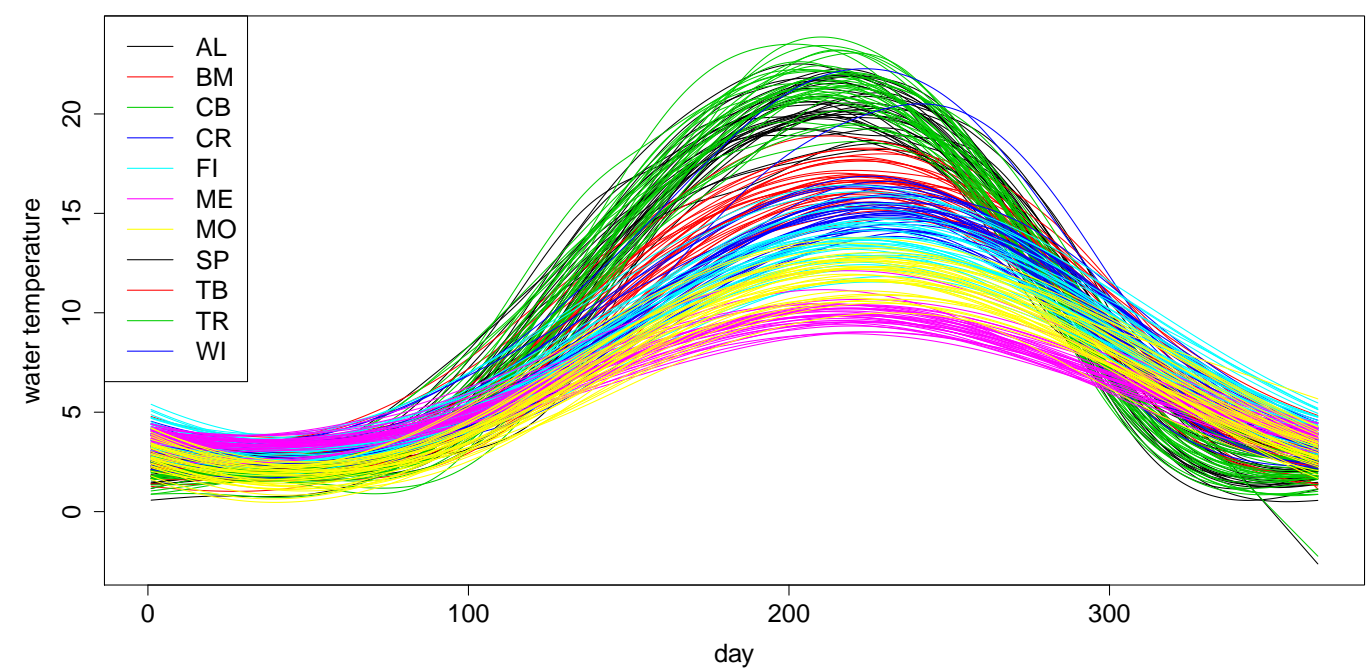

Figure 10: Water temperature profiles over 7 experimental lakes and 35 years. Copepod populations were modeled as a function of the previous 60 days water temperature.

Plots 11 through 18 repeat the top four panels in Figure 4 for each species from the copepod study. For each species, we provide plots of $g(s)$ (top left), $\beta(t)$ (top right), and $g^{\prime \prime}(s)$ (bottom left) along with pointwise confidence intervals. All these plots are given at the values which minimize GCV. The bottom right of each figure plots $\delta$ as a function of both $\lambda_{g}$ and $\lambda_{\beta}$. Regions where $t_{\lambda_{g}, \lambda_{\beta}}$ exceed the critical value are indicated by a white background and a black square gives the minimizing value of GCV. 

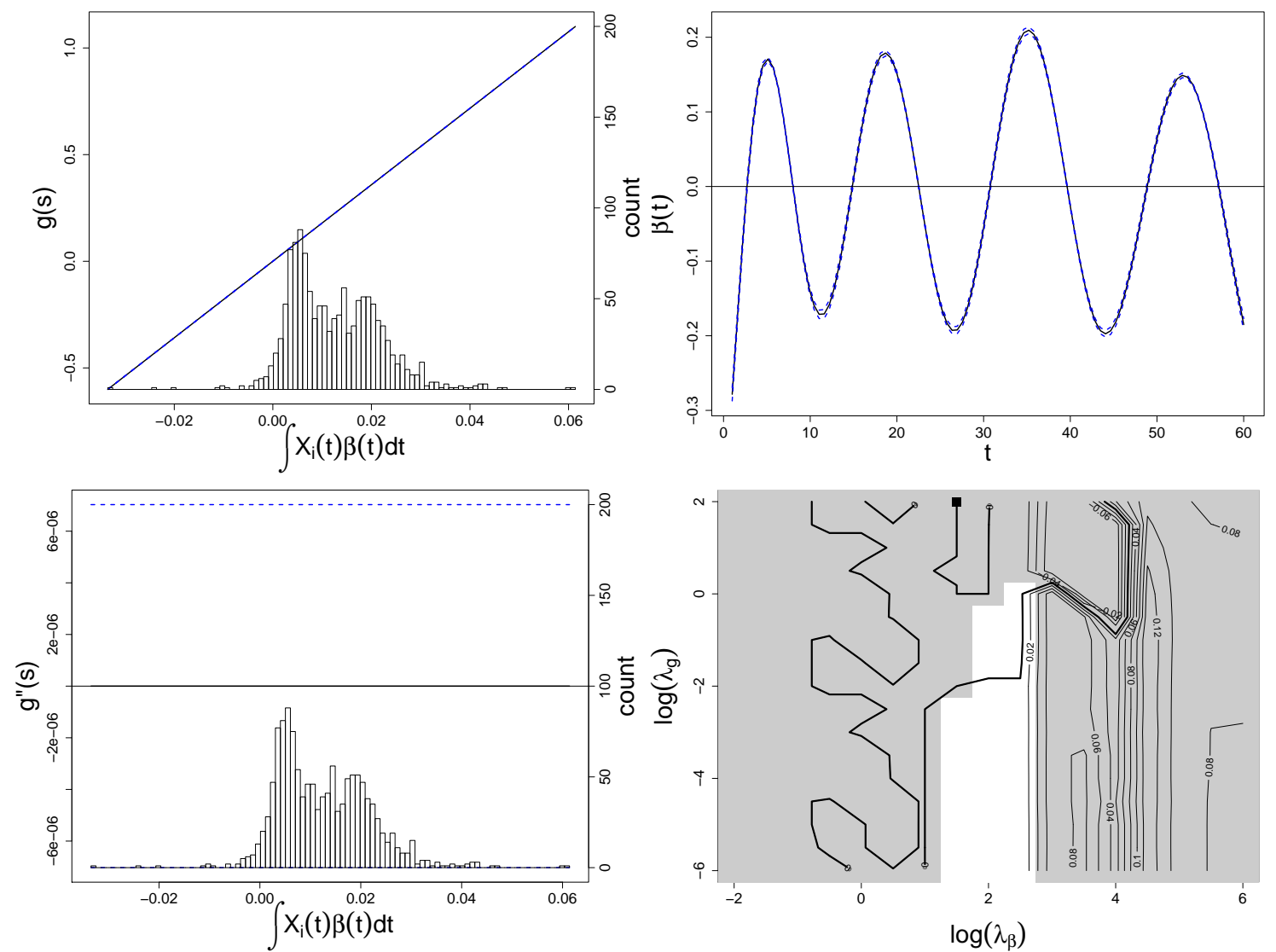

Figure 11: Diagnostic plots for Diacyclops Thomasi, $n=1301$. The region of significance includes negative values (top) and positive values (bottom) with a range of [-0.079, 0.1362]. See dicussion in Section 5 for this species. 

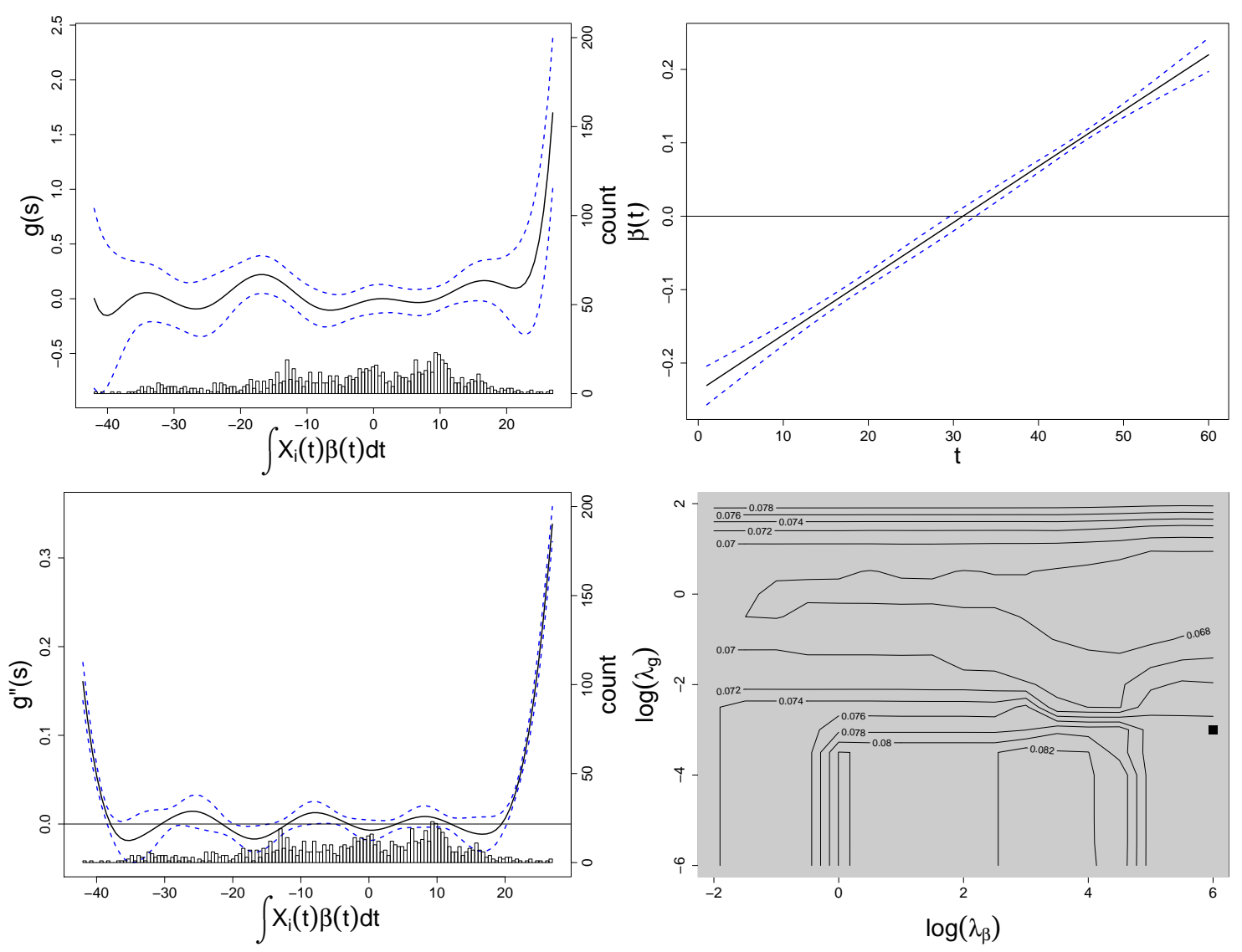

Figure 12: Plots for Gastropus Stylifer, $n=893, \delta$ is estimated to be in the range [0.0663, 0.0830] but is nowhere significant. 

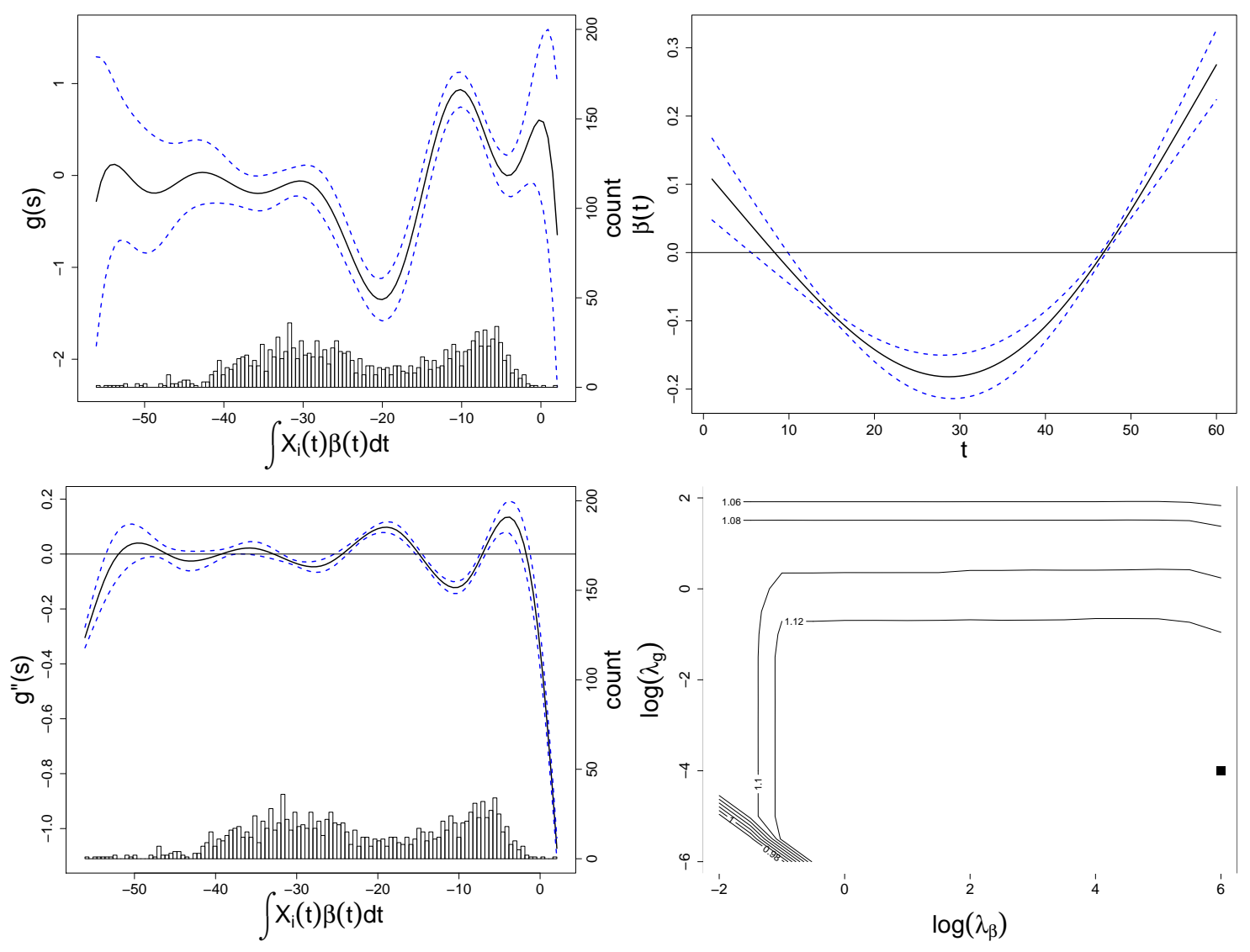

Figure 13: Plots for Kellicottia Longispina, $n=1347 . \delta$ is positive and significant everywhere with values in $[0.970,1.132]$, but note that the largest values occur at the smallest values of the smoothing parameters and may be over-estimates. 

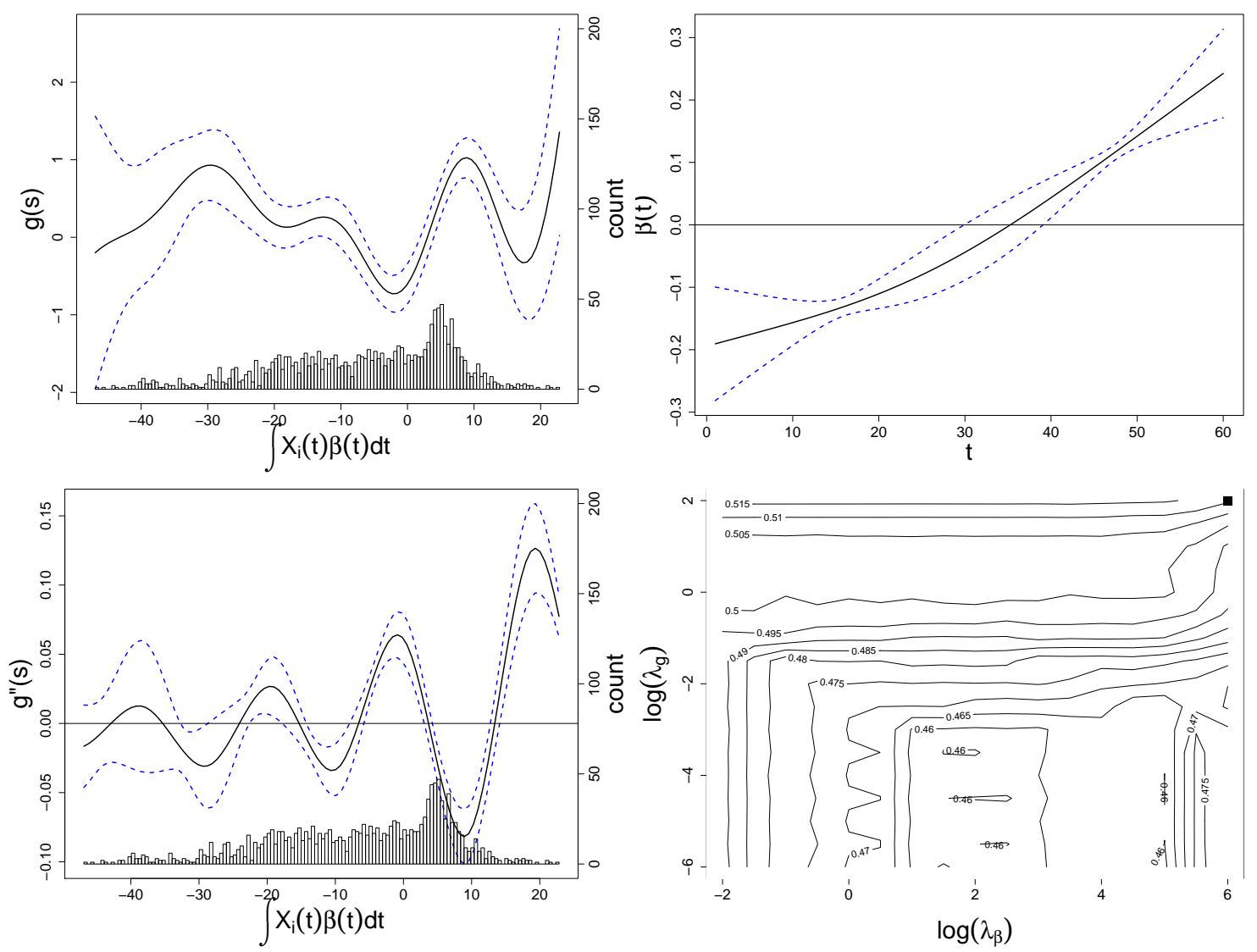

Figure 14: Plot for Keratella Cochlearis, $n=1467$. $\delta$ significant everywhere and in the range [0.458, $0.516]$. 

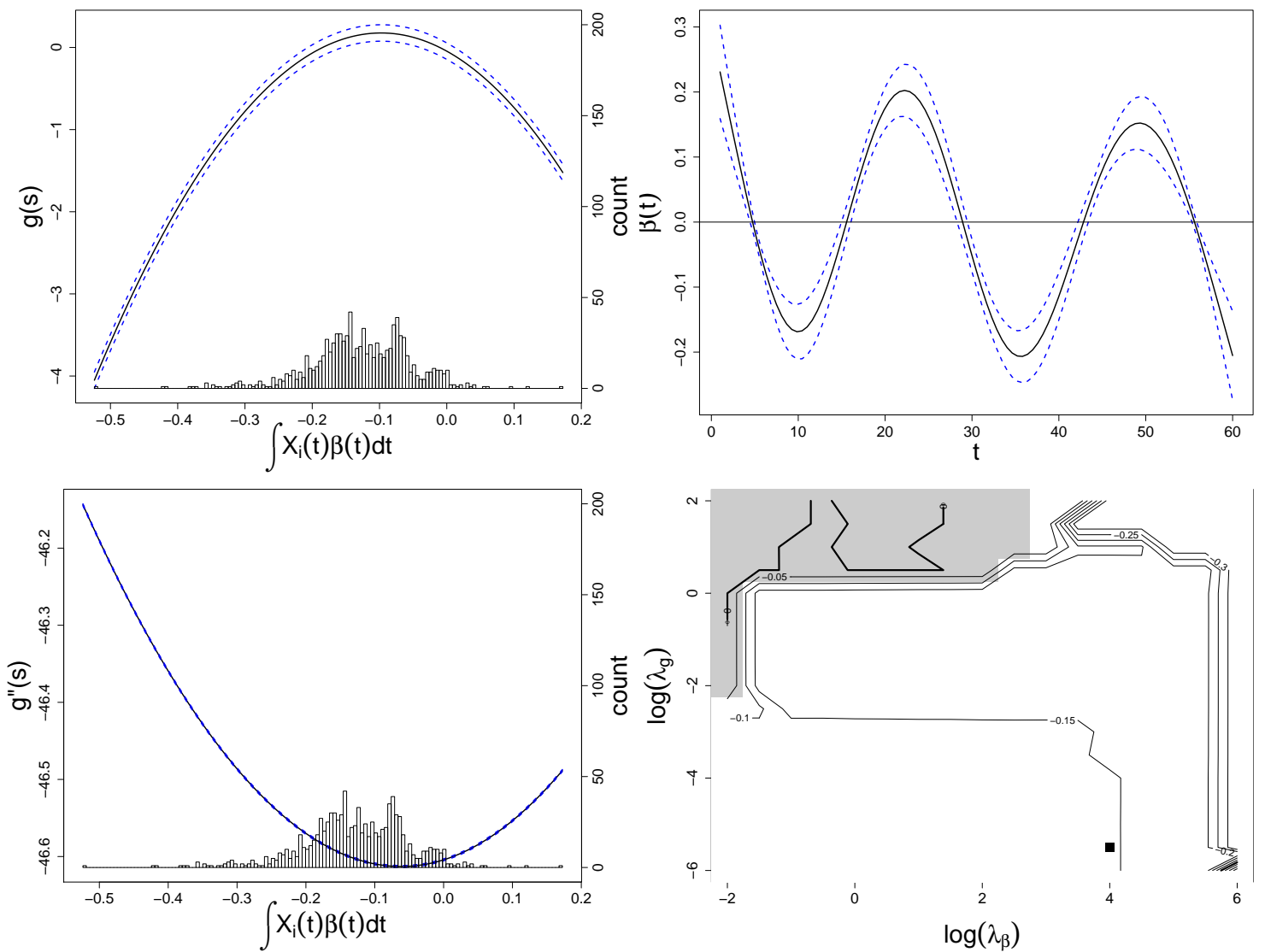

Figure 15: Plot for Keratella Earlinae, $n=946 . \delta$ takes values in the range [-0.348, 0.191]. but note that negative values only occur in the bottom right corner of the contour plot. 

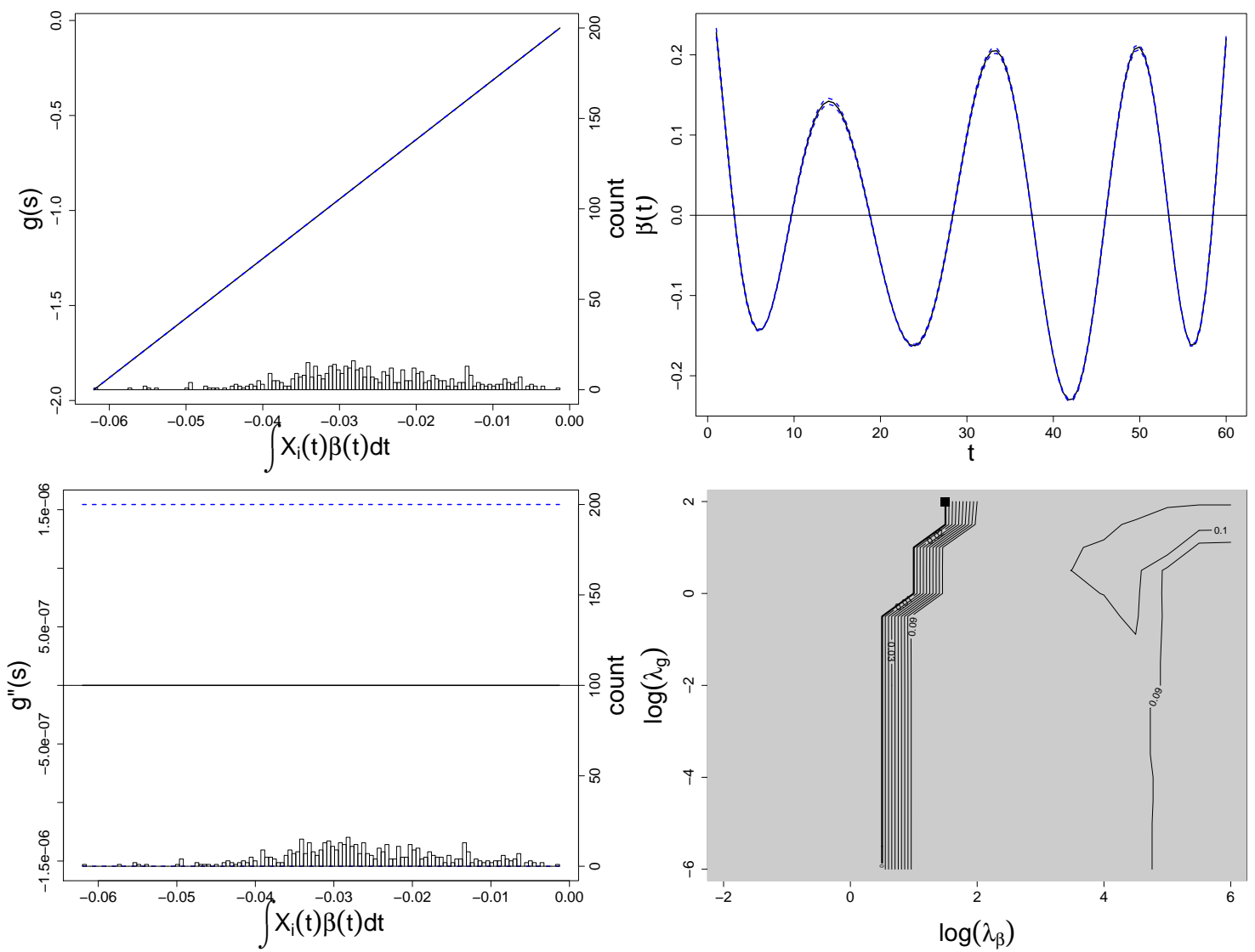

Figure 16: Plot for Keratella Quadrata, $n=521 . \delta$ is estimated in the range $[0,0.1]$ and is nowhere significant. 

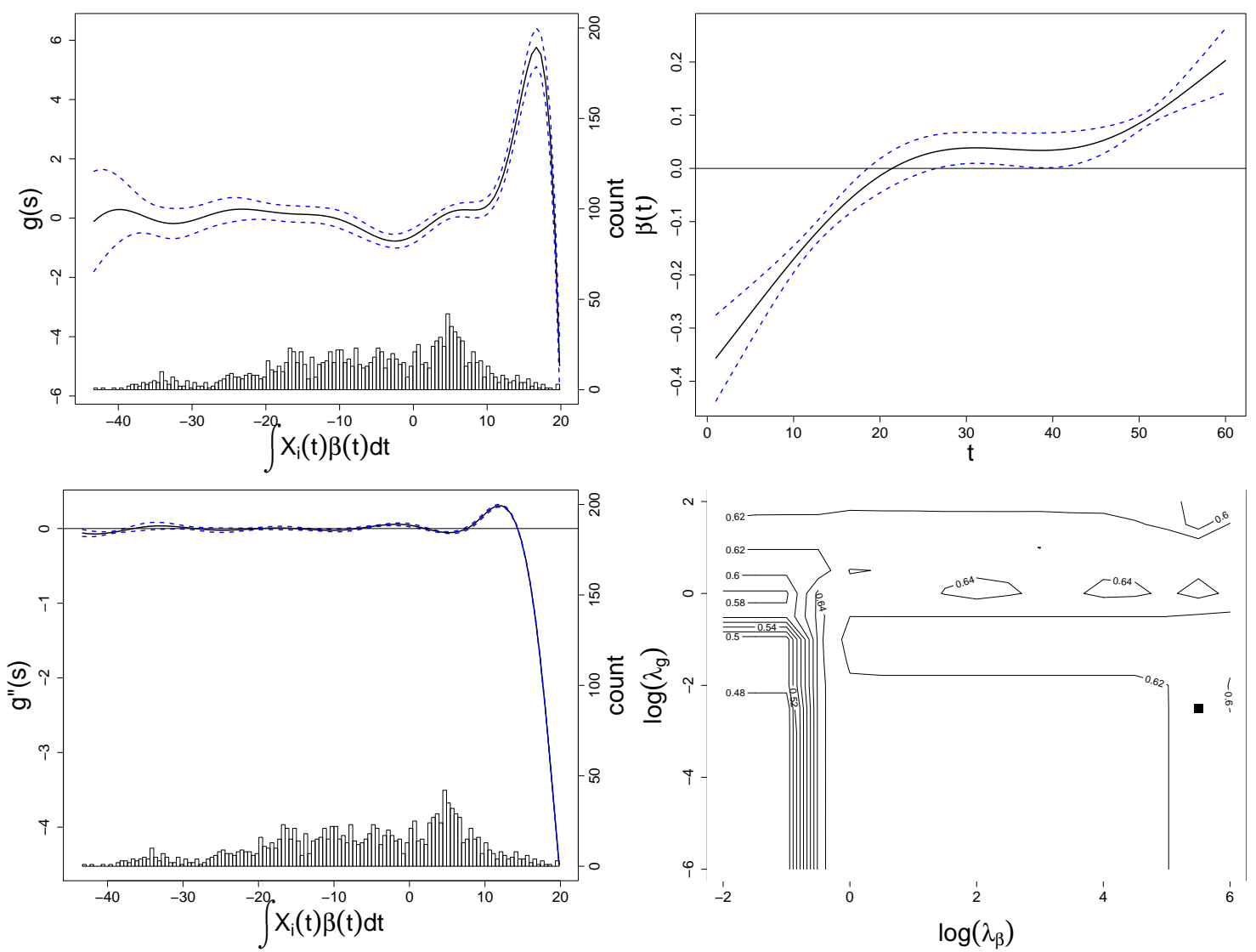

Figure 17: Plot for Polyarthra Remata, $n=1347 . \delta$ takes values in the range [0.462, 0.647]. 

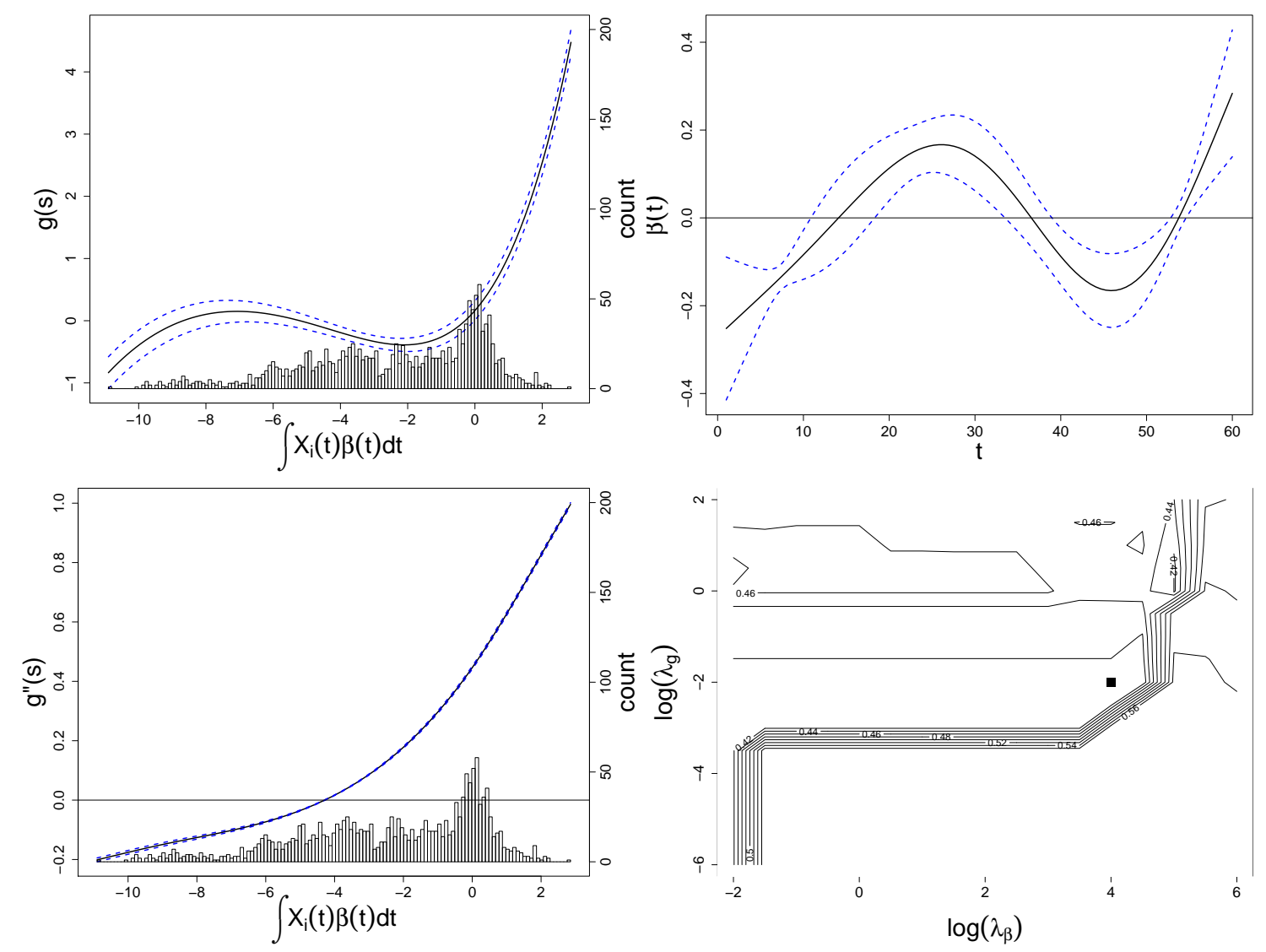

Figure 18: Plot for Polyarthra Vulgaris, $n=1577 . \delta$ takes values in the range $[0.4023,0.5763]$ and is signifcant at all smoothing parameters.

\section{References}

Ye, Z. and G. Hooker (2018). Local quadratic estimation of the curvature in a functional single index model. arXiv preprint arXiv:1803.09321. 